\title{
Die Entwicklung der Entschädigung und der reisebezogenen Ansprüche im deutschen Abgeordnetenrecht
}

\author{
Philipp Austermann*
}

Die Mitglieder des Deutschen Bundestages und der Landesparlamente erhalten, wie die Mitglieder aller demokratischen Parlamente weltweit, ein Einkommen aufgrund des Mandats. Dieses wird im Fachdeutsch zumeist „Entschädigung“ und im Volksmund „Diäten“ genannt. Daneben steht den deutschen Abgeordneten eine (je nach Parlament unterschiedliche) sogenannte Amtsausstattung aus finanziellen und Sachmitteln zu. Zumeist besitzen sie außerdem reisebezogene Ansprüche: eine Freifahrtberechtigung und/oder ein Recht auf Reisekostenersatz. Die rechtliche Entwicklung der finanziellen Leistungen für Abgeordnete ist ein guter Indikator für die im Laufe der Jahrzehnte fortschreitende Professionalisierung der Abgeordnetentätigkeit in der Bundesrepublik Deutschland.

\section{Die finanzielle Ausstattung des Mandats: Bedingung für das passive Wahlrecht}

Die finanziellen Leistungen für Abgeordnete, vornehmlich der Entschädigungsanspruch, aber auch die reisebezogenen und weiteren Ansprüche erscheinen heute selbstverständlich. Allerdings mussten das Entschädigungs- und das Freifahrtrecht regelrecht erstritten werden. Beispielsweise dauerte es im Deutschen Reich von dessen Gründung im Jahr 1871 an 35 Jahre, bis die „Reichsleitung“ und der Bundesrat ihr Einverständnis gaben, den Abgeordneten durch zwei Reichsgesetze vom 21. Mai $1906^{1}$ eine vergleichsweise geringe Entschädigung zu gewähren. ${ }^{2}$ Das Freifahrtrecht erhielten die Reichstagsmitglieder schon 1874, doch wurde es zeitweise wieder ausgesetzt. ${ }^{3}$ Beide Rechte sind große demokratische Errungenschaften. Die den Lebensunterhalt sichernde Abgeordnetenentschädigung ist eine notwendige tatsächliche und rechtliche Bedingung für das passive Wahlrecht; und die Freifahrtberechtigung stärkt das passive Wahlrecht, denn sie ermöglicht es jedem, trotz der dazu erforderlichen und zum Teil erheblichen Reisekosten ein Mandat zu übernehmen.

Aus den bescheidenen Anfängen des Entschädigungs- und des Freifahrtsanspruchs hat sich in Deutschland im Laufe der Zeit und vor allem in der zweiten Hälfte des 20. Jahrhunderts ein ausdifferenzierter Katalog an abgeordnetenrechtlichen Ansprüchen auf finanzielle beziehungsweise geldwerte Leistungen herausgebildet. Die Geschichte dieser Entwicklung stellt der folgende Beitrag dar.

* Der Verfasser ist Referent in der Verwaltung des Deutschen Bundestages; er gibt in diesem Beitrag ausschließlich seine persönliche Meinung wieder.

1 RGBl. S. 467, S. $468 \mathrm{ff}$.

2 Siehe zu den Einzelheiten der Entwicklung Hermann Butzer, Diäten und Freifahrt im Deutschen Reichstag, Düsseldorf 1999; Philipp Austermann, Die Geschichte der Abgeordnetenentschädigung, in: Suzanne S. Schüttemeyer / Edzard Schmidt-Jortzig (Hrsg.), Der Wert der Repräsentation. Entwicklungslinien und Perspektiven der Abgeordnetenentschädigung, Baden-Baden 2014, S. $103-145$.

3 Siehe dazu Hermann Butzer, a.a.O. (Fn. 2), S. $103 \mathrm{ff}$. 


\section{Entschädigungsanspruch ${ }^{4}$}

\subsection{Bundesrecht}

Gemäß Art. 48 Absatz 3 Satz 1 GG haben die Bundestagsabgeordneten Anspruch auf eine angemessene, ihre Unabhängigkeit sichernde Entschädigung. Das „Nähere“, die Einzelheiten, regelt gemäß Satz 3 ein Bundesgesetz. Seit dem sogenannten Diätenurteil des Bundesverfassungsgerichts vom 5. November 1975 ist klargestellt, dass die Entschädigung eine Bezahlung der Abgeordneten aufgrund ihres Mandats, ein (steuerpflichtiges) „Mandatsgehalt", darstellt. ${ }^{5}$ Der Entschädigungsanspruch aus Art. 48 Abs. 3 Satz 1 GG umfasst nicht nur ein monatliches Gehalt, sondern auch einen Anspruch auf begrenztes Übergangsgeld sowie auf eine begrenzte Alters- und Hinterbliebenenversorgung. ${ }^{6}$

Die Staatspraxis und die Staatsrechtslehre der frühen Bundesrepublik knüpften an hergebrachte Sichtweisen aus der Kaiserzeit und der Weimarer Republik an: Das Mandat wurde weiterhin als Ehrenamt und die Abgeordnetenentschädigung dementsprechend als vorveranschlagte oder pauschalierte Aufwandsentschädigung verstanden. ${ }^{7}$ Das erste Gesetz über die Entschädigung der Mitglieder des Bundestages („Diätengesetz“) ${ }^{8}$ trat am 15. Juni 1950 in Kraft. Es legalisierte rückwirkend die innerhalb des Zeitraums vom 1. September 1949 bis zum 15. Juni 1950 ohne Rechtsgrundlage erbrachten Leistungen an die Bundestagsabgeordneten. ${ }^{9}$

Auffallend ist die Kontinuität bestimmter Regelungen, die sich schon während der Weimarer Republik (jedenfalls im Gesetz über die Entschädigung der Mitglieder des Reichstags vom 15. Dezember $1930^{10}$ ) und zum Teil schon im Entschädigungsgesetz vom 21. Mai $1906^{11}$ fanden. $\$ 1$ des Entschädigungsgesetzes von 1950 sprach, wie auch seine Vorgängernormen, von der „Aufwandsentschädigung“. Diese betrug gemäß $\$ 1$ Abs. 1 Nr. 2 monatlich 600 DM (1930: 600 RM) und war zum Monatsersten im Voraus zu zahlen. Sie sollte die mit der Übernahme des Mandats verbundenen wirtschaftlichen Nachteile ausglei-

4 Die folgende Passage zum Entschädigungsanspruch ist - leicht verändert und mit freundlicher Genehmigung des Nomos Verlages, Baden-Baden - dem Beitrag von Philipp Austermann, a.a.O. (Fn. 2), S. $134-142$, entnommen.

5 Vgl. BVerfGE 40, S. 296, S. 314.

6 Zu den Einzelheiten Philipp Austermann, Die Anrechnungsbestimmungen im Abgeordnetenrecht des Bundes und der Länder, Bern 2011, S. 95 ff.

7 Vgl. zum Beispiel Friedrich Giese, Verfassungsrechtlicher Status des Abgeordneten im Verfassungsstreit, in: AöR, 81. Jg. (1956), S. 104 - 108, S. 107; Bruno Schmidt-Bleibtreu / Franz Klein, Kommentar zum Grundgesetz für die Bundesrepublik Deutschland, Neuwied / Berlin 1967, Art. 48, Rn. 7; anderer Ansicht Hermann von Mangoldt / Friedrich Klein, Das Bonner Grundgesetz, Bd. II, München 1964, Art. 48 IV 1; gegen eine Einordnung als bloßes Ehrenamt auch der damalige Bundestagspräsident Eugen Gerstenmaier, BT-PIPr. 3/23, S. 1249. Zur Auffassung der Kaiserzeit Julius Hatschek, Das Parlamentsrecht des Deutschen Reiches, 1. Teil, Berlin 1915, S. 571 f., S. 614 ff.; zur herrschenden Auffassung während der Weimarer Zeit Gerhard Anschütz, Die Verfassung des Deutschen Reichs vom 11. August 1919, Berlin 1933, Art. 40, Anm. 2.

8 BGBl. S. 215.

9 So der Abgeordnete (und Berichterstatter) Hugo Scharnberg bei der Begründung des Gesetzentwurfes, BT-PIPr., 1. WP, S. 2121.

10 RGBl. II S. 1275.

11 RGBl. S. 468. 
chen. ${ }^{12}$ Die Entschädigung war - wie bereits in der Kaiserzeit und während der Weimarer Republik - steuerfrei. ${ }^{13}$ Auch den schon traditionellen Abzug für das Fernbleiben gab es weiterhin: Für unentschuldigte Abwesenheit bei einer Plenarsitzung oder namentlichen Abstimmung wurden pro Tag 20 DM, also 1/30 der Monatssumme ${ }^{14}$, von der Entschädigung abgezogen (vgl. $\$ 3$ Abs. 1). Auch die Regeln für Abgeordnete mit zwei Parlamentsmandaten (Doppelmandatare), die Unverzichtbarkeit und Unübertragbarkeit in $\$ 4$ und $\$ 7$ erinnern an die entsprechenden Bestimmungen des Entschädigungsgesetzes von 1930 (und in Vorgängernormen). Mit $\$ 2$ existierte auch eine Tagegeldregelung, die über die Vorgängervorschriften hinausging und die zum Bezug berechtigenden Anlässe ausweitete: Abgeordnete erhielten ein Tagegeld in Höhe von 30 DM, wenn sie an den Sitzungen des Plenums, eines Ausschusses, einer Fraktion oder weiteren im Gesetz genannten Terminen teilnahmen. Das Tagegeld diente der Unterkunft und Verpflegung in Bonn. Für An-, Abreise- und Überbrückungstage war die Höhe des Tagegeldanspruchs nach der Entfernung zwischen dem Wohnort und Bonn gestaffelt. Wer im Umkreis von $50 \mathrm{~km}$ um Bonn wohnte, erhielt für diese Tage kein Tagegeld. Eine grundlegende Neuerung im Vergleich zur kaiserzeitlichen beziehungsweise Weimarer Rechtslage war die Erstattung von Kosten in Ausübung des Mandats („Unkosten“) gemäß $₫ 5$. Insoweit sah $₫ 1$ der Ausführungsbestimmungen, die der Bundestagspräsident im Benehmen mit dem Ältestenrat am 20. Juni 1950 erließ, einen Pauschalbetrag für mandatsbedingte Aufwendungen wie Büro- und Telefonkosten im Wahlkreis, Büromaterialien, Porti etc. sowie gegen Nachweis einen darüber hinausgehenden Betrag bis zur Höhe von 200 DM vor. ${ }^{15}$

In jeder der ersten sechs Wahlperioden wurde ein neues Entschädigungsgesetz erlassen (1950, 1954, 1958, 1964, 1968) beziehungsweise das bestehende geändert (1961). ${ }^{16}$ Durch $\$ 1$ Satz 1 des Entschädigungsgesetzes von 1958 wurde die Entschädigung an die Bundesministerbesoldung gekoppelt. Sie betrug 22,5 Prozent des Amtsgehalts.

Schrittweise wurden neue Leistungsarten eingeführt: im Jahr 1954 das Übergangsgeld und das Sterbegeld für die Hinterbliebenen (vgl. $\$ 1$ Abs. 3 Satz 4 beziehungsweise $\$ 8$ des Entschädigungsgesetzes von 1954) ${ }^{17}$ und 1968 eine Alters- und Hinterbliebenenversorgung auf Versicherungsbasis ${ }^{18}$ sowie eine Invalidenversorgung (vgl. $\$ 4$ des Entschädigungsgesetzes von 1968 beziehungsweise $\$ 8$ des „DiätenG 1968“). Die Abgeordneten hatten 25 Prozent ihrer monatlichen Entschädigung als Beiträge zur Alters- und Hinterbliebenenversicherung abzuführen. Die monatlichen Bezüge wurde daher auf 3,33 Prozent der Bun-

12 Vgl. Hugo Scharnberg, a.a.O. (Fn. 9), S. 2120.

13 Vgl. nur Hermann von Mangoldt / Friedrich Klein, a.a.O. (Fn. 8), Art. 48 IV 3 a; Bruno SchmidtBleibtreu / Franz Klein, a.a.O. (Fn. 8), Art. 48, Rn. 8.

14 Vgl. Hugo Scharnberg, a.a.O. (Fn. 9), S. 2121.

15 Entsprechend (dem Vorschlag) der Anlage zum Mündlichen Bericht des Haushaltsausschusses vom 31. März 1950 über den Entwurf eines Gesetzes über die Entschädigung der Mitglieder des Bundestages, BT-Drs. 1/797, S. 7.

16 Gesetz vom 24. Juni 1954 (BGBl. II S. 637); Gesetz vom 27. Mai 1958 (BGBl. I S. 379); Gesetz vom 15. Juni 1961 (BGBl. I S. 763); Gesetz vom 25. März 1964 (BGBl. I S. 230); Gesetz vom 3. Mai 1968 (BGBl. I S. 334); letzteres geändert durch die Gesetze vom 22. Juni 1972 (BGBl. I S. 993), vom 2. September 1974 (BGBl. I S. 2151), vom 24. Juni 1975 (BGBl. I S. 1599), vom 18. August 1976 (BGBl. I S. 2195) und vom 18. Februar 1977 (BGBl. I S. 308).

17 Beide Ansprüche wurden durch das Gesetz vom 15. Juni 1961 inhaltlich ausgebaut.

18 Zur Vorgeschichte, auch in den Ländern, Hartmut Klatt, Die Altersversorgung der Abgeordneten, Tübingen 1972, S. 17 ff. 
desministerbesoldung erhöht (vgl. $\$ 1$ Satz 2). ${ }^{19}$ Die Anrechnung anderweitiger öffentlichrechtlicher Einkünfte auf die Alters- und Hinterbliebenenversorgung wurde gesetzlich ausgeschlossen (vgl. \$10).

Der Ersatz mandatsbedingter Aufwendungen über Tagegelder, Unkosten- sowie Reisekostenerstattung wurde ausgeweitet. Das Entschädigungsgesetz von 1958 schrieb jeweils eine monatliche Pauschalzahlung fest (vgl. $\$ 2, \$ 3$ und $\$ 5$ ).

Eine Unfallversicherung der Bundestagsabgeordneten durch das Parlament war seit 1950 möglich und seit 1958 Pflicht.

Das mit den Jahren erweiterte Leistungsspektrum entsprach der tatsächlichen Veränderung der Abgeordnetentätigkeit. Es wurde immer deutlicher, dass diese nicht einfach als ehrenamtliche (Neben-)Tätigkeit (ohne Alters- und Hinterbliebenenversorgung) aufgefasst werden konnte. Schon der Erste Bundestag erledigte trotz der vergleichsweise einfachen Arbeitsbedingungen - für 402 Abgeordnete standen nur 50 Büros zur Verfügung - ein enormes Pensum. ${ }^{20}$ Auch in den folgenden Wahlperioden bewährte sich der Bundestag als Arbeitsparlament, das die sozialen und wirtschaftlichen Herausforderungen annahm. Das Bundesverfassungsgericht stellte in seiner Entscheidung vom 16. März 1955 fest, dass „im Zuge der in einer Reihe von Staaten zu beobachtenden Entwicklung von der liberal-repräsentativen zur parteienstaatlichen Demokratie [...] in der politisch-gesellschaftlichen Wirklichkeit auch der Charakter der Aufwandsentschädigung sich allmählich zu wandeln“ scheine und ,in einigen Staaten sich mehr und mehr einem Entgelt für die im Parlament geleisteten Dienste" annähere. ${ }^{21}$ In der Entscheidung vom 21. Oktober $1971 \mathrm{zu} \$ 12$ Abs. 1 und 2 des Hessischen Abgeordnetengesetzes merkte es an, dass sich die Abgeordnetenentschädigung „mehr und mehr einem Entgelt für die im Parlament geleisteten Dienste angenähert [habe] und mehr und mehr den Charakter einer Besoldung oder eines Gehalts“22 annehme. Zum ersten Mal wurde der Ruhegehaltsanspruch als Annex der Entschädigung angesehen. ${ }^{23}$ In seinem Urteil vom 5. November 1975, dem „Diätenurteil“, stellte das Bundesverfassungsgericht dann einen Verfassungswandel fest: Die Bezüge seien von einer Entschädigung für ein Ehrenamt zu einer Bezahlung, einem Gehalt, für die im Parlament geleistete Tätigkeit geworden. ${ }^{24}$ Von diesem Standpunkt ist das Gericht bis heute zu Recht nicht abgewichen. Die ganz herrschende Meinung im staatsrechtlichen Schrifttum hat sich ihm angeschlossen. ${ }^{25}$

Nach dem Diätenurteil hat der Bund sein Abgeordnetenrecht neu kodifiziert. An die Stelle des DiätenG 1968 trat im Jahr 1977 das Gesetz zur Neuregelung der Rechtsverhältnisse der Mitglieder des Deutschen Bundestages, kurz Abgeordnetengesetz (AbgG). Es un-

19 So der Abgeordnete (und Berichterstatter) Ulrich Berger, BT-PIPr., 5. WP, S. 8503.

20 Vgl. Hans-Peter Schwarz, Gründerjahre der Republik, Stuttgart 1981, S. 86.

21 BVerfGE 4, S. 144, S. 151.

22 BVerfGE 32, S. 157, S. 164.

23 Vgl. BVerfGE 32, S. 157, S. 165; bestätigt in BVerfGE 40, S. 296, S. 311.

24 Vgl. BVerfGE 40, S. 296, S. 314.

25 Vgl. nur Hans Hugo Klein, in: Theodor Maunz / Günter Dürig / Roman Herzog / Rupert Scholz, Grundgesetz, Loseblatt (Stand: Mai 2013), Art. 48, Rn. 115 ff.; Hermann Butzer, in: Volker Epping / Christian Hillgruber (Hrsg.), Grundgesetz, Berlin 2013, Art. 48, Rn. 14 mit weiteren Nachweisen; der herrschenden Meinung mit gewissen Abweichungen zustimmend Philipp Austermann, a.a.O. (Fn. 6), S. 78 ff. 
terscheidet zwischen (nunmehr) steuerpflichtigen ${ }^{26}$ alimentativen Leistungen und der steuerfreien Aufwandsentschädigung. Zu den ersteren gehören - während der Mandatszeit - die Grundentschädigung ( $\$ 11$ spricht wenig differenzierend von der „Abgeordnetenentschädigung "), also das monatliche Abgeordnetengehalt, und die Erstattung von Kosten in Krankheits-, Pflege- und Geburtsfällen (\$27), sowie - unmittelbar nach dem Mandatsende - das Übergangsgeld ( $\$ 18)$ und - beim Erreichen der maßgeblichen Altersgrenze - die Altersversorgung („Altersentschädigung“, $\$ \$ 19,20$ AbgG). Die Höhe des Altersversorgungsanspruchs richtet sich seitdem nach einem Prozentsatz der Abgeordnetenentschädigung pro Jahr der Mitgliedschaft im Deutschen Bundestag. Sie steigt also mit der Anzahl der Mandatsjahre. Da das BVerfG die Koppelung der Grundentschädigung an ein Beamtengehalt in einem obiter dictum ${ }^{27}$ des Diätenurteils für unzulässig erklärte ${ }^{28}$, sah (und sieht) das Abgeordnetengesetz die Koppelung der Grundentschädigung an die Bundesministerbezüge nicht mehr vor. Stattdessen steht seitdem der konkrete Betrag der monatlichen Grundentschädigung im Gesetz ( $\$ 11 \mathrm{AbgG})$. Anderweitige Einkünfte öffentlich-rechtlicher und zum Teil auch privatrechtlicher Herkunft werden auf die Abgeordnetenbezüge angerechnet $(\$ 18$ Abs. 2, \$29 AbgG). Die dem Bundestag noch in $\$ 11$ DiätenG 1968 vorgeschriebene Unfallversicherung wurde mit dem Inkrafttreten des Abgeordnetengesetzes abgeschafft.

Die Aufwandsentschädigung wird in $\$ 12$ Abs. 1 AbgG als Amtsausstattung bezeichnet. Zur Amtsausstattung gehören zunächst die Bereitstellung eines eingerichteten Büros am Sitz des Bundestages, die Bereitstellung und Nutzung des gemeinsamen Informations- und Kommunikationssystems, die Benutzung der Dienstfahrzeuge („Fahrbereitschaft“) sowie sonstige Leistungen; sodann ist der Reisekostenersatz gemäß $\$ 16$ AbgG ${ }^{29}$ Teil der Amtsausstattung ( $\$ 12$ Abs. 4 AbgG). Zudem werden die Kosten für die Beschäftigung von Mitarbeitern ersetzt (vgl. $\$ 12$ Abs. 3 AbgG). Außerdem erhalten die MdBs eine steuerfreie Kostenpauschale, zum Beispiel für die Unterkunft in Berlin, Wahlkreisbüros oder Fahrten mit dem PKW im Wahlkreis (vgl. $\$ 12$ Abs. 2 AbgG). Bis zum Inkrafttreten des Abgeordnetengesetzes erhielten die Abgeordneten zum Ersatz mandatsbedingter Aufwendungen die Tagegeld- und die Unkostenpauschale ${ }^{30}$ sowie eine Reisekostenpauschale (vgl. \$ 13 DiätenG 1968) und gemäß $\$ 17$ DiätenG 1968 die Erstattung gewisser Reisekosten gegen Nachweis. Die drei Pauschalen verschmolzen zur Kostenpauschale; die Reisekostenerstattung gegen Nachweis lebt in $\$ 16$ AbgG fort.

Wenngleich das Abgeordnetengesetz in seinen Grundzügen seit dem Jahr 1977 unverändert geblieben ist, wurde es im Detail fast dreißig Mal geändert ${ }^{31}$, nicht zuletzt, weil aus

26 Vgl. die Materialien zum Entwurf eines Gesetzes zur Neuregelung der Rechtsverhältnisse der Mitglieder des Deutschen Bundestages, BT-Drs. 7/5531, S. 8, S. 18 (zu $\$ 13$ AbgG-E, dem späteren $\$ 11 \mathrm{AbgG})$.

27 Vgl. nur Brun-Otto Bryde, Verfassungsentwicklung. Stabilität und Dynamik im Verfassungsrecht der Bundesrepublik Deutschland, Baden-Baden 1982, S. 171.

28 Vgl. BVerfGE 40, S. 296, S. 316 f.

29 Siehe dazu Näheres im Abschnitt 3.

30 Seit 1972 nur noch „Kostenpauschale“, vgl. den Entwurf eines Gesetzes zur Änderung des Gesetzes über die Entschädigung der Mitglieder des Bundestages vom 1. Februar 1972, BT-Drs. 6/3092, S. 1, S. 4.

31 Vgl. Zehntes Gesetz zur Änderung des Parteiengesetzes und Achtundzwanzigstes Gesetz zur Änderung des Abgeordnetengesetzes vom 23. August 2011 (BGBl. I S. 1748), Berichtigung vom 20. Dezember 2011 (BGBl. I S. 3141). 
(verfassungsrechtlich nicht zwingenden) Transparenzerwägungen der exakte Betrag der Abgeordnetenentschädigung im Gesetz verankert ist (vgl. \$11 Abs. 1 AbgG). Jede Anpassung der Entschädigung bedeutet eine Änderung des Abgeordnetengesetzes (wenngleich die Erhöhung in mehreren Schritten nach zutreffender Ansicht in einem Änderungsgesetz geregelt werden kann).

Das Neundzwanzigste Änderungsgesetz ${ }^{32}$ (das bei Drucklegung noch nicht in Kraft getreten war) hat der Deutsche Bundestag am 21. Februar 2014 verabschiedet. ${ }^{33}$ Der Bundesrat hatte in seiner 920. Sitzung am 14. März 2014 keine Einwände. ${ }^{34}$ Das Gesetz geht auf den Bericht der Unabhängigen Kommission zu Fragen des Abgeordnetenrechts vom 19. März 2013 zurück. ${ }^{35}$ Es bringt - neben Änderungen etwa bei der Höhe des Altersversorgungsanspruchs - zwei Neuerungen: Erstens wird das Anpassungsverfahren für die Abgeordneten(grund)entschädigung auf eine sogenannte Indexierung umgestellt (vgl. \$ 11 Abs. 4 AbgG n. F.). ${ }^{36}$ Dafür wird der vom Statistischen Bundesamt erhobene Bruttonominallohnindex verwendet, der die Entwicklung der Einkommen der abhängig Beschäftigten im Bundesgebiet angibt. Wenn also die Einkommen nach diesem Index beispielweise um 2,5 Prozent gestiegen sind, steigt die Abgeordnetenentschädigung in derselben Höhe. Wenn umgekehrt die Einkommensentwicklung im Vorjahr negativ verlief, sinkt die Entschädigung entsprechend. Die neue Höhe der Bezüge ist vom Bundestagspräsidenten in einer Bundestagsdrucksache zu veröffentlichen. Die Indexierung greift erstmals zum 1. Juli 2016. Ausgangsgröße der Indexierung ist gemäß $\$ 11$ Abs. 1 Satz 1 AbgG n. F. die Besoldung eines Richters an einem obersten Gerichtshof des Bundes (Besoldungsgruppe R 6 gemäß der Anlage IV des Bundesbesoldungsgesetzes mit Zulage für Richter und Staatsanwälte bei obersten Gerichtshöfen des Bundes). Da die Entschädigung sich zwar schon seit dem Jahr $1995^{37}$ - wie $\$ 11$ Abs. 1 AbgG (a. F.) zeigt - ausdrücklich an der Besoldungsgruppe R 6 und B 6 orientieren sollte, diese Richtgrößen aber bislang noch nie erreicht hat ${ }^{38}$, wird sie - vor dem Start des Indexierungsverfahrens im Jahr 2016 - in zwei Schritten auf das Niveau der genannten Richterbesoldung angehoben und ab dem 1. Januar 2015 monatlich 9.082 Euro betragen (vgl. $\$ 11$ Abs. 1 Satz 2 AbgG n. F.). Das beschriebene Indexierungsverfahren bleibt gemäß $\$ 11$ Abs. 5 AbgG n. F. für eine neue Wahlperiode nur wirksam, wenn der Bundestag innerhalb von drei Monaten nach der konstituierenden Sitzung einen entsprechenden Beschluss fasst. Geschieht dies nicht, gilt für die Entschädigung der letzte per Index ermittelte Betrag, bis der Bundestag das Anpassungsverfahren in einem Gesetz bestätigt oder ändert.

Die zweite Neuerung des Neunundzwanzigsten Änderungsgesetzes besteht darin, dass neben dem Präsidenten und den Vizepräsidenten des Deutschen Bundestages nunmehr auch die Vorsitzenden von ständigen Ausschüssen, Untersuchungsausschüssen und En-

32 Vgl. den Entwurf eines $x x$. Gesetzes zur Änderung des Abgeordnetengesetzes und eines $x x$. Gesetzes zur Änderung des Europaabgeordnetengesetzes vom 11. Februar 2014, BT-Drs. 18/477.

33 Vgl. BT-PIPr. 18/18, S. 1371 ff., S. 1388.

34 Vgl. BR-Pr. der 920. Sitzung, S. 28.

35 BT-Drs. 17/12500; siehe dazu auch den Beitrag von Edzard Schmidt-Jortzig in diesem Heft der ZParl.

$36 \mathrm{Zu}$ den Gründen siehe BT-Drs. 18/477, S. 10 f.

37 Gesetz zur Neuregelung der Rechtsstellung der Abgeordneten vom 15. Dezember 1995 (BGBl. I S. 1718).

38 Vgl. BT-Drs. 18/477, S. 7. 
quetekommissionen eine Funktionsvergütung aus dem Bundeshaushalt erhalten (vgl. $\$ 11$ Abs. 2 AbgG n. F.). ${ }^{39}$

\subsection{Landesrecht}

In allen Landesverfassungen, mit Ausnahme der saarländischen, findet sich eine Bestimmung zur Abgeordnetenbezahlung. Zumeist sind die Formulierungen dem Art. 48 Abs. 3 GG ähnlich (vgl. etwa Art. 40 der Verfassung des Landes Baden-Württemberg, Art. 11 Abs. 3 der Verfassung des Landes Schleswig-Holstein). Der Wortlaut einiger vor dem Grundgesetz erlassenen Verfassungen knüpft noch an das frühere Entschädigungsverständnis an: Sie sprechen von der „Aufwandsentschädigung“ (vgl. Art. 31 der Verfassung des Freistaates Bayern) oder sogar von „Sitzungsgeldern“ sowie einer „Aufwandsentschädigung“ für den Landtagspräsidenten (vgl. Art. 98 Abs. 1 der Verfassung des Landes Hessen). Die Verfassungen der norddeutschen Stadtstaaten gehen modernere Wege und haben den Begriff „Entschädigung“ durch „Entgelt“ ersetzt (vgl. Art. 13 Abs. 1 Satz der Verfassung der Freien und Hansestadt Hamburg, Art. 82 Abs. 2 der Landesverfassung der Freien Hansestadt Bremen). Die Freifahrtberechtigung besitzt in fünf Ländern Verfassungsrang. Einige Verfassungen gewähren auch Ansprüche auf den Ersatz mandatsbedingter Aufwendungen (vgl. etwa Art. 54 Abs. 3 der Verfassung des Freistaats Thüringen). Hinsichtlich der Einzelheiten, des „Näheren“, verweisen fast alle Verfassungen auf ein (Landes-)Gesetz.

Vor dem Diätenurteil wurden auch die Mandate in Landesparlamenten von der weit überwiegenden Meinung als Ehrenamt verstanden. Die Abgeordnetenbezüge sollten demnach eine (steuerfreie) Aufwandsentschädigung sein. ${ }^{40}$ Seit dem Diätenurteil werden die Verfassungsbestimmungen der Länder, unabhängig davon, ob sie die „Entschädigung“ erwähnen oder andere Formulierungen gebrauchen, im Sinne eines Einkommens (einer Alimentation) aufgrund der Mandatstätigkeit verstanden. ${ }^{41}$ Bei der Ausgestaltung (Anspruchsvoraussetzungen, Bezugsdauer, Höhe) besitzen die Länder einen weiten Spielraum. Sie können unter anderem der Arbeitsbelastung der Abgeordneten beziehungsweise dem Selbstverständnis ihrer Volksvertretung als Vollzeit- oder Teilzeitparlament Rechnung tragen. Allerdings umfassen die verfassungsrechtlichen Entschädigungsansprüche neben einem monatlichen Gehalt auch einen Anspruch auf begrenztes Übergangsgeld sowie auf eine begrenzte Alters- und Hinterbliebenenversorgung. ${ }^{42}$

Die Abgeordnetengesetze der Länder wurden seit dem Jahr 1977 in Anlehnung an das Abgeordnetengesetz des Bundes ausgestaltet. Neben die Grundentschädigung als monatliches Gehalt traten Aufwandsentschädigungen, zum Beispiel eine Kostenpauschale oder die Erstattung von Kosten für Mitarbeiter. Für die unmittelbare Zeit nach dem Ausscheiden aus dem Parlament wurde ein Übergangsgeld vorgesehen. Alle Bundesländer führten zu-

39 Zu den Gründen siehe BT-Drs. 18/477, S. 9 f.

40 Vgl. zum Beispiel Hans Herbert von Arnim, Die Mär vom Landtagsmandat als Fulltimejob, in: ZRP, 38. Jg. (2005), S. 77 - 78; für das frühere Verständnis in Schleswig-Holstein Hans-Jochen Waack, in: Johannes Caspar / Wolfgang Ewer / Martin Nolte / ders. (Hrsg.), Verfassung des Landes Schleswig-Holstein, Kiel 2006, Art. 11, Rn. 44 ff.

41 Vgl. Philipp Austermann, a.a.O. (Fn. 6), S. 140 mit weiteren Nachweisen.

42 Vgl. ebenda, S. $145 \mathrm{ff}$. 
dem eine beamtenrechtsähnliche Altersversorgung ein. Die landesrechtlichen Regelungen wichen und weichen im Detail von der Bundesregelung, aber auch untereinander ab. Generell folgten die Länder aber den vielen kleineren Änderungen im Bundesrecht, zum Beispiel bei den Verschärfungen der Anrechnungsbestimmungen.

Seit der deutschen Einheit ist eine weitergehende Reformtätigkeit im Abgeordnetenrecht der Länder erkennbar. Den ersten Schritt ging Thüringen, das die Indexierung der Abgeordnetenentschädigung in Art. 54 seiner Verfassung verankerte. Einige Bundesländer haben die beamtenrechtsähnliche Altersversorgung abgeschafft: Nordrhein-Westfalen besitzt seit dem Jahr 2005 ein Versorgungswerk für die Abgeordneten, an dem sich mit Beginn der 6. Wahlperiode seines Landtages im Jahr 2014 auch Brandenburg beteiligen wird. Schleswig-Holstein, Bremen und Baden-Württemberg setzen auf eine private Eigenvorsorge, die aus vom Parlament während der Mandatszeit gewährten Vorsorgebeiträgen und damit (hoffentlich) zu erzielenden Zinserträgen basiert. Sachsen besitzt - je nach Wahl des Abgeordneten - ein „Mischsystem“ aus beamtenrechtsähnlicher Altersversorgung und Eigenvorsorge. Auch die steuerfreie Aufwandsentschädigung ist einem Wandel unterworfen. Die genannten Länder - mit Ausnahme Sachsens - haben sie radikal gekürzt oder ganz gestrichen und dafür im Gegenzug die steuerpflichtige Grundentschädigung stark erhöht.

\section{Reisebezogene Ansprüche (Freifahrtberechtigung und Reisekostenerstattung)}

\subsection{Bundesrecht}

Die Bundestagsabgeordneten haben gemäß Art. 48 Absatz 3 Satz 2 GG das Recht der freien Benutzung aller staatlichen Verkehrsmittel. Art. 48 Abs. 3 Satz 2 GG meint mit den „staatlichen Verkehrsmitteln“ nur solche des Bundes und - anders als Art. 40 der Weimarer Reichsverfassung vom 11. August 1919 (WRV) ${ }^{43}$ - nicht solche der Länder, der Gemeinden oder Privater. ${ }^{44}$ Entscheidend ist, dass der Träger des Verkehrsmittels (wie noch die Deutsche Bahn AG oder früher die Lufthansa AG) unter dem rechtlichen Einfluss des Bundes steht. ${ }^{45}$ Ein solcher Einfluss ist bei einem Anteil von mehr als 50 Prozent gegeben. ${ }^{46}$ Aktuell lässt sich aus der Verfassung ein Anspruch nur noch für die Verkehrsmittel der Deutschen Bahn AG herleiten (vgl. auch $\$ 16$ Abs. 1 Satz 1 AbgG). Über Art. 48 Abs. 3 Satz 2 GG hinausgehende Ansprüche, etwa in Bezug auf die Nutzung anderer Verkehrsmittel oder Übernachtungskosten, sind gemäß Art. 48 Abs. 3 Satz 3 GG als „das Nähere“ durch Bundesgesetz zu regeln.

Das oben bereits erwähnte erste Gesetz über die Entschädigung der Mitglieder des Bundestages vom 15. Juni 1950 regelte - rückwirkend seit dem 1. September 1949 - in $\$ 1$ Abs. 1 Nr. 1 die Freifahrtberechtigung. Der Regelungsinhalt der Norm stimmte im We-

43 Art. 40 WRV gab den Mitgliedern des Reichstages das Recht zur freien Fahrt auf allen deutschen Eisenbahnen. Somit waren auch Privatbahnen erfasst, vgl. Gerhard Anschütz, a.a.O. (Fn. 7), Art. 40, Rn. 1.

44 Vgl. statt vieler Hans Hugo Klein, a.a.O. (Fn. 25), Art. 48, Rn. 193.

45 Vgl. nur Hermann Butzer, a.a.O. (Fn. 25), Art. 48, Rn. 25; Walter Georg Leisner, in: Helge Sodan (Hrsg.), Grundgesetz, Berlin 2011, Art. 48, Rn. 10.

46 Vgl. Hermann Butzer, a.a.O. (Fn. 25), Art. 48, Rn. 25. 
sentlichen mit der Vorgängernorm aus der Weimarer Zeit, $\$ 1$ Abs. 1 Nr. 1 des Gesetzes über die Entschädigung der Mitglieder des Reichstags vom 15. Dezember 1930 ${ }^{47}$, überein: Die Mitglieder des Bundestages erhielten für die Dauer ihrer Zugehörigkeit zum Bundestag und die folgenden acht Tage, im Falle einer Neuwahl des Bundestages jedoch bis zum Ablauf des achten Tages nach der Wahl des neuen Bundestages, das Recht der freien Benutzung aller Verkehrsmittel der Deutschen Bundesbahn und der Deutschen Post. Durch die neu eingeführte und im Rahmen des Entschädigungsanspruchs bereits erwähnte Unkostenpauschale waren unter anderem die Kosten für die Nutzung eines Autos abgedeckt, ohne dass dies in der Norm Erwähnung fand. Der Gesetzentwurf sah noch eine explizite Regelung vor ${ }^{48}$, von der aber im Zuge der Plenarberatungen abgesehen wurde. ${ }^{49}$ Stattdessen wurde die Kostenerstattung durch $\$ \$ 2$ f. der Ausführungsbestimmungen geregelt, die der Bundestagspräsident gemäß $₫ 5$ im Benehmen mit dem Ältestenrat erließ. ${ }^{50}$ Durch $\$ 4$ Satz 2 wurde - wie zuvor durch $₫ 5$ Satz 2 des Entschädigungsgesetzes von 1930 - ein Fahrtkostenersatz durch eine andere öffentlich-rechtliche Körperschaft ausgeschlossen.

Durch das Entschädigungsgesetz von $1958^{51}$ wurde das bislang auf die Freifahrtberechtigung beschränkte Leistungsspektrum um eine monatliche Reisekostenpauschale $(\$ 6)$ und den Kostenersatz für Dienstreisen $(\$ 7)$ erweitert. Durch die Zahlung der Reisekostenpauschale galten gemäß $\$ 6$ Abs. 1 Satz 3 alle Unkosten als abgegolten, die den Abgeordneten für Fahrten im Wahlkreis und im Raum Bonn, durch die Benutzung von Schlafwagen, Flugzeugen und Kraftwagen, einschließlich der Reisekosten für Kraftfahrer, entstanden. Die Reisekostenpauschale war in gewissem Umfang eine Ausgliederung aus der Unkostenpauschale, etwa bezüglich des Aufwands für die Automobilnutzung. Die Höhe der Reisekostenpauschale bemaß sich gemäß $\$ 6$ Abs. 1 Sätze 1 und 2 nach den Entfernungen zwischen Wohnsitz und Sitz des Bundestages, wobei die Pauschalsätze nach Zonen gestaffelt waren. Die in Wahlkreisen direkt gewählten Abgeordneten konnten die Pauschale entsprechend der Entfernung zwischen dem Sitz des Bundestages und dem Amtssitz des jeweiligen Kreiswahlleiters in Anspruch nehmen ( $\$ 6$ Abs. 2). Die Pauschalsätze wurden durch Ausführungsbestimmungen festgelegt, die der Bundestagspräsident im Benehmen mit dem Ältestenrat erließ (\$ 6 Abs. 1 Satz 4).

Die Kategorie der Dienstreise war ebenfalls neu. Sie setzte die Zustimmung des Präsidenten voraus ( $\$ 7$ Satz 1 ) und konnte den Abgeordneten sowohl ins In- als auch ins Ausland führen. Nur bei Auslandsreisen erhielten Abgeordnete gemäß $₫ 7$ Satz 2 Tagegelder (nach bestimmten beamtenrechtlichen Regelungen) sowie die „Fahrtkosten 1. Klasse“ von der Bundesgrenze bis zum Tagungsort und zurück. An Sitzungstagen wurde das Dienstreisetagegeld gekürzt. In bestimmten Fällen wurden auch Schlafwagen-, Flug- oder Schiffsreisekosten erstattet (vgl. $\$ 7$ Sätze 5 bis 8$)$.

47 RGBl. II S. 1275.

48 Vgl. $\$ 5$ des Gesetzentwurfes vom 23. März 1950, BT-Drs. 1/704, S. 3.

49 Vgl. die Ausführungen des Abgeordneten (und Berichterstatters) Hugo Scharnberg bei der Begründung des Gesetzentwurfes, BT-PIPr., 1. WP, S. 2120, und den Mündlichen Bericht des Haushaltsausschusses über den genannten Gesetzentwurf vom 31. März 1950, BT-Drs. 1/797, S. 5.

50 Entsprechend dem Vorschlag, der der Anlage zum Mündlichen Bericht des Haushaltsausschusses vom 31. März 1950 über den Entwurf eines Gesetzes über die Entschädigung der Mitglieder des Bundestages, BT-Drs. 1/797, S. 7, zu entnehmen ist.

51 Vgl. Fn. 16. 
Die geschilderte Struktur aus Freifahrtanspruch, Reisekostenpauschale und Kostenersatz für Dienstreisen blieb auch nach den Entschädigungsgesetzen von 1964, 1968 und dem Änderungsgesetz von $1972^{52}$ erhalten. Es kam aber zu kleineren Änderungen: Beispielsweise konnte gemäß $₫ 8$ Abs. 1 Satz 1 des Entschädigungsgesetzes von 1964 nun auch bei Inlandsdienstreisen Übernachtungsgeld (in entsprechender Anwendung der beamtenrechtlichen Regelungen) gezahlt werden. Durch das Diätengesetz von 1968 verlor der Freifahrtanspruch seinen prominenten Platz im ersten Paragraphen, den er seit dem Entschädigungsgesetz von 1906 innegehabt hatte. Er war bis 1958 sogar stets vor der Entschädigung genannt worden. Nunmehr wurde die Freifahrtberechtigung erst in $\$ 17$ Abs. 1 Satz 1 DiätenG 1968 erwähnt. Neu eingeführt wurde die Kostenerstattung gegen Nachweis für die Nutzung von Flugzeugen und Schlafwagen in Ausübung des Mandats im Bundesgebiet ( $\$ 17$ Abs. 1 Satz 2 DiätenG 1968). Beides wurde aus der Reisekostenpauschale herausgenommen. Die Rechtsänderung verfolgte das Ziel, die allgemein übliche Benutzung von Flugzeugen und Schlafwagen auch den MdBs zu ermöglichen. Diese diene, so die Begründung, der Zeitersparnis und Arbeitserleichterung, die angesichts der ständig steigenden Inanspruchnahme der Parlamentarier in und außerhalb Bonns geboten und gerechtfertigt sei. ${ }^{53}$ Anzumerken ist, dass die Nutzung dieser Verkehrsmittel schon vorher möglich war. Die Kosten dafür waren aber aus der Reisekostenpauschale zu bestreiten, wie $\$ 6$ des Entschädigungsgesetzes von 1964 zeigt. Die Dienstreiseregelung in $\$ 19$ erhielt einen Verweis auf das wenige Jahre zuvor erlassene Bundesreisekostengesetz.

Das Abgeordnetengesetz des Jahres 1977 brachte gewisse Änderungen der geschilderten Struktur und des Leistungsumfangs mit sich. Die Freifahrtberechtigung und der Kostenersatz für Dienstreisen blieben im bekannten Umfang erhalten: $\$ 16$ AbgG knüpfte an $\$ 17$ DiätenG 1968 an, $\$ 17$ AbgG an $\$ 19$ DiätenG 1968. ${ }^{54}$ Die Reisekostenpauschale wurde mit der Kosten- und der Tagegeldpauschale zur „neuen“ Kostenpauschale zusammengefasst. Die $\$ \$ 16$ und 17 AbgG wurden seit dem Inkrafttreten des Abgeordnetengesetzes bis heute inhaltlich nur wenig verändert. So wurde zum Beispiel nach der Privatisierung der Deutschen Post und der Deutschen Bahn erstere als Verkehrsmittelträger aus dem Abgeordnetengesetz gestrichen. Ferner wurde $\$ 16$ Abs. 1 Satz 2 AbgG durch das 27. Gesetz zur Änderung des Abgeordnetengesetzes vom 2. Dezember $2007^{55}$ dahingehend ergänzt, dass auch die Kosten für „sonstige schienengebundene Beförderungsmittel außerhalb des öffentlichen Personennahverkehrs", also für Privatbahnen ${ }^{56}$, gegen Nachweis ersetzt werden.

$52 \mathrm{Zu}$ allen vgl. ebenda.

53 Vgl. den Schriftlichen Bericht des Vorstandes des Deutschen Bundestages über den von den Fraktionen der CDU/CSU, SPD, FDP eingebrachten Entwurf eines Gesetzes zur Änderung und Ergänzung des Diätengesetzes 1964 - V/2526 - [und] über den von den Abgeordneten Heinrich Windelen, Wilhelm Rawe, Rembert van Delden, Christian Haase, Dr. Wolfgang Rutschke und Genossen eingebrachten Entwurf eines Gesetzes über die Entschädigung der Mitglieder des Bundestages (Diätengesetz 1968) - V/2575 - vom 21. März 1968, BT-Drs. 5/2754, S. 4.

54 Vgl. Werner Braun / Monika Jantsch / Elisabeth Klante, Abgeordnetengesetz des Bundes - unter Einschluß des Europaabgeordnetengesetzes, Berlin 2002, $₫ 16$, Rn. 2 beziehungsweise $₫ 17$, Rn. 2; Materialien zum Entwurf eines Gesetzes zur Neuregelung der Rechtsverhältnisse der Mitglieder des Deutschen Bundestages, BT-Drs. 7/5531, S. 23 ( $\$ 29$ und $₫ 30$ AbgG-E wurden $\$ 16$ und $\$ 17 \mathrm{AbgG}$ ).

55 BGBl. I S. 3212.

56 Vgl. den Entwurf eines Siebenundzwanzigsten Gesetzes zur Änderung des Abgeordnetengesetzes vom 6. November 2007, BT-Drs. 16/6924, S. 9. 
Zusammengefasst sieht die reisebezogene Amtsausstattung der Mitglieder des Bundestages derzeit wie folgt aus: Bundestagsabgeordnete besitzen eine Freifahrtberechtigung für die Verkehrsmittel der Deutschen Bahn AG $^{57}$ (gemäß Art. 48 Abs. 3 Satz 2 GG i. V. m. \$16 Abs. 1 Satz 1 AbgG), von bestimmten Voraussetzungen abhängige und der Höhe nach beschränkte Ansprüche auf Fahrtkostenersatz und Dienstreisekostenersatz (gemäß den $\$ \$ 16$, 17 AbgG) sowie das Recht zur Nutzung der Dienstfahrzeuge des Bundestages (der sogenannten Fahrbereitschaft) im Rahmen der vorhandenen Kapazitäten (gemäß $₫ 12$ Abs. 4 Satz 1 Nr. 2 AbgG). Mandatsbedingte Fahrten mit dem PKW sind wie weitere, nicht von den $\$ \$ 16,17$ AbgG abgedeckte Reisekosten nicht erstattungsfähig, sondern gemäß $\$ 12$ Abs. 2 Nr. 3 AbgG aus der Kostenpauschale zu bestreiten.

Die über mehrere Jahrzehnte erfolgte Erweiterung der reisebezogenen Amtsausstattung hat vier Gründe. Erstens ist die verfassungsrechtliche Aufgabe der Bundestagsabgeordneten zu nennen. Sie sind nicht nur Wahlkreisgesandte, sondern gemäß Art. 38 Abs. 1 Satz 2 GG Vertreter des ganzen Volkes. Daher müssen sie sich auch in ganz Deutschland informieren können. Beispielsweise muss ein Verkehrspolitiker sich nicht allein um seinen Wahlkreis oder sein Heimatbundesland kümmern, sondern muss sich sämtliche Themengebieten, die auf der politischen Tagesordnung seines Ausschusses stehen, widmen. Zweitens ist das erweiterte Leistungsspektrum durch die tatsächliche Veränderung der Abgeordnetentätigkeit, aber auch durch die verbesserten Reisemöglichkeiten begründet. Mussten Abgeordnete in den 1950er und 1960er Jahren zum Teil äußerst zeitraubende Reisen in die Bundeshauptstadt unternehmen, können sie nun durch die Nutzung von Flugzeugen ihre Effizienz steigern. Drittens sind mit den verbesserten Reisemöglichkeiten auch die Erwartungen der Öffentlichkeit gestiegen, dass Abgeordnete leichter erreichbar und vor Ort verfügbar sind. Viertens sind die Außenbeziehungen der Bundesrepublik Deutschland und ihres wichtigsten Verfassungsorgans, des Deutschen Bundestages, seit 1949 stetig gewachsen und vertieft worden. Gerade seit der Wiedervereinigung hat sich die internationale Rolle Deutschlands verändert. Die Verantwortung für das Weltgeschehen ist gestiegen. Deutschland beteiligt sich im Rahmen seiner Bündnisverpflichtungen an bewaffneten Auslandseinsätzen. Es ist zudem stark eingebunden in die Europäische Union. Abgeordnete müssen sich informieren und die Regierungstätigkeit im In- und Ausland kontrollieren. Dies alles (erforderte und) erfordert Reisen sowie eine dementsprechende Amtsausstattung.

\subsection{Landesrecht}

Nur fünf Landesverfassungen sehen einen Freifahrtanspruch - innerhalb der jeweiligen Landesgrenzen - vor (vgl. Art. 31 BayVerf, Art. 40 Satz 2 BWVerf, Art. 98 Abs. 1 Satz 1 HessVerf, Art. 50 Satz 1 NWVerf, Art. 42 Abs. 3 Satz 2 SächsVerf). Die übrigen Länder kennen nur einfachgesetzliche Freifahrtregelungen, die auf der Verfassungsermächtigung zur Regelung des „Näheren“ per Parlamentsgesetz basieren. Sonstiger Reisekostenersatz, etwa für Flug- oder Übernachtungskosten, ist stets einfachgesetzlich geregelt. Nur Art. 98 Abs. 1 Satz 1 HessVerf kennt einen verfassungsunmittelbaren Anspruch auf Reisekostenersatz, ohne aber die Details zu normieren.

57 Die Jahresnetzkarte wird von der Bundestagsverwaltung erworben und an die Abgeordneten ausgegeben. 
Die Geschichte der reisebezogenen Ansprüche ist derjenigen im Bund vergleichbar. Die Länder haben in der Regel Rechtsentwicklungen im Bund - auf ihre Verhältnisse angepasst - zeitverzögert nachvollzogen. Die dabei erfolgte Ausweitung des Leistungsspektrums ist auch in den Ländern ein Zeichen der Professionalisierung der Abgeordnetentätigkeit. Allerdings unterscheidet sie sich doch in einigem von der Tätigkeit der Mitglieder des Deutschen Bundestages. Sie stellt in der Regel zeitlich geringere Anforderungen und ist auf einen begrenzteren Wirkungskreis gerichtet als das Bundestagsmandat. Die Entfernungen zwischen Wohnort und Parlamentssitz sind in der Regel signifikant geringer als zwischen dem jeweiligen Wohnort und Berlin. Auch tagen die Landesparlamente und ihre Ausschüsse seltener als der Bundestag und dessen Gremien. Zudem ist die Pflege der Beziehungen zu auswärtigen Staaten gemäß Art. 32 Abs. 1 GG Sache des Bundes, weshalb die Länder bei weitem nicht so ausgeprägte Auslandsbeziehungen wie der Bund unterhalten. Aus allen genannten Gründen ist der Umfang der reisebezogenen Ansprüche in den Ländern zwangsläufig stets (deutlich) geringer als im Bund.

Bestimmte Grundprinzipien sind allen Ländern mehr oder weniger gemeinsam. Gleichwohl waren und sind die Einzelheiten, also die rechtstechnische Umsetzung, die Anspruchsvoraussetzungen und die Erstattungshöhe sowie die Reichweite der Freifahrtberechtigung, unterschiedlich. Im Rahmen dieser kurzen Dokumentation kann nur ein grober Überblick über die Entwicklung und die derzeitige Rechtslage gegeben werden. Zunächst kannten die Entschädigungsgesetze (Abgeordnetengesetze) der Länder wie dasjenige des Bundes nur die Freifahrtberechtigung als reisebezogenen Anspruch. Im Laufe der Zeit traten dann, wie im Bund, der Kostenersatz für Dienstreisen und (in den meisten Ländern) der Kostenersatz für sonstige Reisen hinzu.

Aktuell wird in fast allen Bundesländern (mit Ausnahme Berlins, Bremens und Schleswig-Holsteins) ein nachweisloses Freifahrtrecht auf öffentlichen Verkehrsmitteln gewährt. Es ist grundsätzlich auf die Nutzung öffentlicher Verkehrsmittel innerhalb des Bundeslandes beschränkt (vgl. etwa Art. 6 Abs. 5 BayAbgG, $\$ 8$ Abs. 1 BbgAbgG). Alle Länder gewähren Dienstreisekostenersatz (in unterschiedlicher Terminologie), der in der Regel von der Zustimmung des Parlamentspräsidenten zu der betreffenden Reise abhängt (vgl. etwa Art. 10 BayAbgG, $\$ 9$ BbgAbgG, $\$ 9$ RhPfAbgG, $\$ 14$ SHAbgG). Zumeist ist auch der Ersatz sonstiger Kosten für Reisen innerhalb des Landes gegen Nachweis vorgesehen (vgl. etwa $\$ 7$ HessAbgG, $\$ \$ 10$ bis 13 MVAbgG). In einigen Ländern werden Reisekosten ganz oder zum Teil über die Kostenpauschale abgedeckt (vgl. etwa Art. 6 Abs. 2 Satz 1 Nr. 2 und 3 BayAbgG, $\$ 6$ Abs. 3 Satz 1 Nr. 3 BbgAbgG, $\$ 6$ Abs. 2 Nr. $3 \$ 9$ RhPfAbgG, $\$ 6$ Abs. 2 SächsAbgG).

\section{Die finanzielle Ausstattung des Mandats als Zeichen seiner Professionalisierung}

An der Entwicklung der Entschädigungshöhe und insbesondere der Ausdifferenzierung in verschiedene Leistungskategorien (Gehalt, Altersversorgung, Kostenpauschale) zeigt sich die im Laufe der Jahrzehnte fortschreitende Professionalisierung der Abgeordnetentätigkeit. Das Mandat ist zu einem Beruf und die Entschädigung zu einem Einkommen aus diesem Beruf geworden. Diese Entwicklung wird auch bei den reisebezogenen Ansprüchen deutlich sichtbar. Aus dem der „Reichsleitung“ und dem Bundesrat im 19. Jahrhundert abgerungenen Freifahrtrecht ist ein Leistungskatalog geworden, der verschiedene mandatsbedingte Not- 
wendigkeiten abdeckt. Zugleich haben die reisebezogenen Ansprüche im Laufe des 20. Jahrhunderts an Bedeutung eingebüßt. War es im 19. Jahrhundert vielen Reichstagsabgeordneten ohne privates Vermögen nicht möglich, an Reichstagssitzungen teilzunehmen, da sie keine Diäten erhielten und während mehrerer Sessionen auch keine Freifahrtberechtigung besaßen, ist deren Wichtigkeit in dem Maße gesunken, wie die Entschädigung erhöht wurde und den Lebensunterhalt zu sichern vermochte. Auch an dieser im Vergleich zu anderen abgeordnetenrechtlichen Leistungen gestiegenen Bedeutung der Entschädigung zeigt sich die fortschreitende Professionalisierung der Abgeordnetentätigkeit.

Das Abgeordnetenrecht des Bundes und der Länder wurde seit der Gründung der Bundesrepublik viele Male modifiziert. Weitere Änderungen werden sicherlich folgen. Die Empfehlungen der Unabhängigen Kommission zu Fragen des Abgeordnetenrechts vom 19. März 2013 haben - wie gezeigt - zu deutlichen Änderungen geführt. Aber auch sie werden wohl nicht die letzten sein. Die häufig allzu erregten Debatten um die Höhe und die Ausgestaltung der finanziellen und geldwerten Leistungen an Abgeordnete gibt es schon seit der Weimarer Zeit. Sie werden mit Sicherheit auch in Zukunft weitergehen. Die Parlamente können sie selbstbewusst führen: Ein angemessenes Einkommen und eine entsprechende Amtsausstattung der Abgeordneten sind nicht Ausdruck irgendeiner Selbstbedienungsmentalität, sondern unverzichtbare Voraussetzungen wirklicher Demokratie und erfolgreicher parlamentarischer Repräsentation. Sie ermöglichen die Teilhabe aller am Staatsgeschehen und sichern die für moderne demokratische Gesellschaften unumgängliche Professionalität des Mandats.

\title{
Unerfreuliche Einblicke in Tiefenschichten deutscher Parlaments- wahrnehmung: Die Kritik an den Vorschlägen der Unabhängigen Kommission zu Fragen des Abgeordnetenrechts
}

\author{
Werner J. Patzelt
}

\section{Zur Leistung der Kommission}

Die im November 2011 vom Ältestenrat des Bundestages eingesetzte „Unabhängige Kommission zu Fragen des Abgeordnetenrechts“ war fleißig. Zwischen Dezember 2011 und März 2013, als sie ihren Bericht dem Bundestagspräsidenten übergab ${ }^{1}$, tagte sie nicht weniger als 17-mal im vertraulichen Kreis ihrer elf Mitglieder, hörte 13 Experten und arbeitete sich, gestützt auf ein vierköpfiges Sekretariat, durch das einschlägige, auch innerstaatlich und international vergleichende rechts- sowie politikwissenschaftliche Schrifttum, durch

1 „Bericht und Empfehlungen der Unabhängigen Kommission zu Fragen des Abgeordnetenrechts“, veröffentlicht als BT-Drs. 17/12500 vom 19. März 2013, ebenso in: Suzanne S. Schüttemeyer / Edzard Schmidt-Jortzig (Hrsg.), Der Wert der parlamentarischen Repräsentation. Entwicklungslinien und Perspektiven der Abgeordnetenentschädigung, Baden-Baden 2013, S. 17 - 102. Eine Einschätzung der Arbeit und Leistung der Expertenkommission aus der Sicht ihres Vorsitzenden findet sich unter anderem in einem Interview mit Edzard Schmidt-Jortzig, in: Das Parlament vom 22. April 2013 sowie in seinem Beitrag in diesem Heft der ZParl. 